Unidad de Tratamiento del

Ataque Cerebrovascular

(UTAC), Servicio de Neurología,

Departamento de Medicina,

Clínica Alemana de Santiago.

Facultad de Medicina Clínica

Alemana-Universidad del

Desarrollo. Santiago, Chile.

Financiamiento: Ninguno

Recibido el 13 de febrero de 2016, aceptado el 30 de agosto de 2016.

Correspondencia a:

Dr. Alejandro Brunser R.

Servicio de Neurología, Clínica

Alemana de Santiago.

Manquehue Norte $1410,10^{\circ}$

piso, Vitacura, Santiago, Chile.

Fax: 56-2-22101061

abrunser2002@yahoo.com

\section{Trombolisis intravenosa en cuadros imitadores de isquemia cerebral: experiencia de un centro}

\author{
ALEJANDRO M. BRUNSER, ARNOLD HOPPE, \\ PAULA MUÑOZ, PABLO M. LAVADOS, SERGIO ILLANES, \\ VIOLETA DÍAZ, VERÓNICA V. OLAVARRÍA
}

\section{Thrombolysis in stroke mimics. Experience in 10 cases}

Background: The effectiveness of intravenous thrombolysis (IVT) in cerebral ischemia is time dependent. Stroke mimics (SM) are frequent in emergency rooms. The effort to reduce door to needle time, can lead to administer thrombolytics to SM. Aim: To describe the frequency and prognosis of SM treated with IVT. Material and Methods: Prospective analysis of all patients evaluated in a Chilean private clinic between December 2004 and July 2015 with a suspected acute ischemic stroke (AIS). We calculated the number of SM that were treated with IVT. In these patients, we analyzed the presence of symptomatic cerebral hemorrhage defined as the presence of a neurological deterioration of four points or more on the National Institute of Health Stroke Scale, the presence of extracranial bleeding according to Global Utilization of Streptokinase and t-PA for Occluded Coronary Arteries criteria and the patient's functional status at three months by modified Rankin scale (MRS). Results: We evaluated 1,417 patients with suspected AIS, of which 240 (16.9\%) were finally diagnosed as SM. A total of 197 patients were treated with IVT, of these 10 (5\%) corresponded to SM. All SM patients treated with thrombolytic drugs were functionally independent at 3 months and showed no bleeding complications. Conclusions: Occasionally SM patients were treated with IVT. This treatment was not associated with bleeding complications and the prognosis of these patients at three months was favorable.

(Rev Med Chile 2016; 144: 1266-1269).

Key words: Stroke; Therapeutics; Thrombolytic Therapy.

\section{L}

a trombolisis intravenosa (TIV) hasta las $4,5 \mathrm{~h}$ de evolución es la principal terapia aprobada para el ataque cerebrovascular isquémico (ACVi) agudo ${ }^{1,2}$. Si bien este tratamiento es capaz de aumentar en $30 \%$ la oportunidad de tener mínimas o ninguna secuela, comparado con el placebo, su efecto es tiempo dependiente, presentando mejor respuesta clínica en la medida que el tiempo hasta su aplicación sea menor ${ }^{3}$. Por cada minuto perdido en una isquemia cerebral se puede perder hasta 1,9 millones de neuronas ${ }^{4}$. Es por esto que se ha propuesto como objetivo de tratamiento en muchos centros hospitalarios un tiempo de puerta aguja (tiempo que transcurre desde que el paciente ingresa a un servicio de urgencia hasta que recibe el bolo de trombolítico intravenoso) menor a $60 \mathrm{~min}^{5}$.

Numerosas enfermedades, como migrañas, epilepsias, cuadros conversivos o tóxico-metabólicos, pueden presentarse en los servicios de urgencia con sintomatología similar a un $\mathrm{ACVi}$, estos cuadros llamados imitadores de isquemia cerebral (CIMIC), pueden representar entre 5 y $30 \%$ de los pacientes catalogados inicialmente 
como que ACVi. ${ }^{6-9}$. El esfuerzo por reducir el tiempo de puerta aguja puede ocasionar que se administre tratamiento TIV a pacientes con CIMIC. Lo anterior ha sido esgrimido por médicos de urgencia para no masificar el uso de la terapia trombolítica, pues su beneficio en pacientes con ACVi puede ser contrarrestado por el riesgo de hemorragia cerebral o extracerebral en pacientes que son portadores de $\mathrm{CIMIC}^{10-11}$.

Nuestro objetivo es describir en nuestro centro terciario el pronóstico y complicaciones hemorrágicas de pacientes portadores de CIMIC que fueron tratados con TIV.

\section{Pacientes y Métodos}

En el servicio de urgencia de Clínica Alemana de Santiago, el procedimiento ante la sospecha de un paciente con un infarto cerebral en tiempo de TIV se encuentra protocolizado, se activa un código ACV e inmediatamente un aparato de tomografía axial computado es dejado libre y el neurólogo de turno acude a la brevedad a la urgencia, examina al enfermo evaluando su déficit neurológico por medio de la escala de ACV del National Institutes of Health (NIHSS), se toman exámenes generales de sangre: recuento globular, TP/INR, TTPK, glicemia, creatinina y el enfermo es sometido, posteriormente, a un protocolo de imágenes estandarizado que consiste en: una tomografía computada (TC) de cerebro sin contraste, una angiografía por tomografía de los vasos cervicales/cerebrales y una difusión cerebral por resonancia nuclear magnética de cerebro. En enfermos alérgicos al medio de contraste la angiografía por tomografía, puede ser reemplazada por una angiografía por resonancia nuclear magnética (RNM) o un Doppler transcraneano. Si el paciente es candidato a TIV, el bolo del trombolítico es administrado generalmente luego de la TC cerebral y antes de la llegada de los resultados de los exámenes de sangre, si no existe sospecha de una coagulopatía.

El diagnóstico de ACVi se basa en la presencia por la historia clínica y el examen físico de elementos típicos de daño vascular cerebral, con signos de infarto en la TC de cerebro o difusión por RNM de cerebro de ingreso o de seguimiento o la presencia de un vaso tapado en el territorio sintomático. Aquellos pacientes que, pese a imáge- nes cerebrales y vasculares normales, presentaban un altamente probable síndrome neurovascular, sin otro diagnóstico alternativo de urgencia, fueron catalogados como $\mathrm{ACVi}^{12}$. El diagnóstico de CIMIC se realizó basado en la presencia de déficit focales neurológicos agudos, que pudieran ser explicados en forma categórica por un cuadro médico distinto a un ACVi y el diagnóstico de isquemia cerebral descartado ${ }^{12}$.

Una hemorragia cerebral sintomática secundaria a TIV se definió como la presencia de una hemorragia cerebral en una TC de cerebro, asociada a un deterioro clínico de $\geq 4$ puntos por la escala de NIHSS durante la evolución del enfermo. Las hemorragias extracraneanas mayores y menores fueron diagnosticadas según criterios del estudio $\mathrm{GUSTO}^{13}$. El estado funcional del paciente fue evaluado a los tres meses usando la escala de Rankin modificada (mRS) ${ }^{14-16}$ en una entrevista médica presencial y ocasionalmente telefónica. Se definió como independiente aquellos pacientes que presentaban un puntaje mRS de 0 a 2 y como dependiente a los pacientes con puntajes de 3 a 5 .

Los datos se obtuvieron del Registro de Enfermedades Cerebrovasculares de Clínica Alemana (RECCA), el cual es prospectivo, consta de consentimiento informado escrito y está aprobado por el Comité Ético Científico de Clínica Alemana de Santiago-Universidad del Desarrollo.

\section{Análisis}

Se calculó el porcentaje de pacientes con cuadros con sospecha de ataques cerebrovasculares isquémicos que resultaron corresponder a CIMIC, el porcentaje de TIV que fueron aplicadas a pacientes con CIMIC y el porcentaje de complicaciones hemorrágicas intracraneanas y extracraneanas en pacientes con CIMIC que recibieron TIV, además, se describe el pronóstico de estos pacientes a los 3 meses medida con mRS.

\section{Resultados}

Entre diciembre de 2004 y julio de 2015, ingresaron consecutivamente al Servicio de Urgencia de Clínica Alemana de Santiago 1.417 pacientes con la sospecha de ACVi, de los cuales, 240 (16,9\% IC 95\%; 15-18,9) correspondieron finalmente a cuadros de CIMIC. En este período se realizaron un total de 197 TIV, lo que correspondió a 13,3\% 
Tabla 1. Características de los pacientes portadores de cuadros imitadores de isquemia cerebral que recibieron trombolítico intravenoso

\begin{tabular}{|c|c|c|c|c|c|c|c|c|}
\hline Paciente & Sexo & $\begin{array}{l}\text { Edad } \\
\text { (años) }\end{array}$ & $\begin{array}{l}\text { Factores } \\
\text { de riesgo }\end{array}$ & NIHSS & $\begin{array}{l}\text { Síntomas de } \\
\text { presentación }\end{array}$ & $\begin{array}{l}\text { Diagnóstico } \\
\text { final }\end{array}$ & $\begin{array}{l}\text { Complicación } \\
\text { hemorrágica }\end{array}$ & $\begin{array}{c}\text { mRS a } \\
3 \text { meses }\end{array}$ \\
\hline 1 & M & 84 & $\begin{array}{c}\text { Hipertensión } \\
\text { arterial }\end{array}$ & 5 & Hemiparesia & $\begin{array}{c}\text { Crisis } \\
\text { convulsiva }\end{array}$ & No & 0 \\
\hline 2 & M & 74 & Tabaco & 12 & Hemiparesia & $\begin{array}{l}\text { Hiponatremia } \\
\text { severa }\end{array}$ & No & 0 \\
\hline 3 & $\mathrm{~F}$ & 27 & Ninguno & 3 & Afasia expresiva & Migraña con aura & No & 0 \\
\hline 4 & $\mathrm{~F}$ & 55 & Tabaco & 3 & Afasia expresiva & Migraña con aura & No & 0 \\
\hline 5 & $\mathrm{~F}$ & 52 & Tabaco & 5 & Hemiparesia & Migraña con aura & No & 0 \\
\hline 6 & M & 69 & Ninguno & 20 & $\begin{array}{c}\text { Afasia } \\
\text { Hemiparesia }\end{array}$ & Encefalitis viral & No & 1 \\
\hline 7 & M & 36 & Ninguno & 7 & Afasia global & Encefalitis viral & No & 0 \\
\hline 8 & M & 38 & Ninguno & 7 & Afasia agloval & Encefalitis viral & No & 0 \\
\hline 9 & M & 66 & HTA & 1 & Ataxia axial & Vértigo & No & 0 \\
\hline 10 & M & 71 & HBP, DLP & 4 & $\begin{array}{l}\text { Hemianopsia } \\
\text { Disartria }\end{array}$ & Tumor cerebral & No & 2 \\
\hline
\end{tabular}

(IC 95\%; 12,2-15,8) del total de casos con sospecha de ACVi. Diez pacientes (5\% IC 95\%; 2,7-9,1) del total que recibió TIV correspondió a cuadros CIMIC, lo que representa a $0,67 \%$ (IC 95\%; 0,38-1,2) del total de enfermos consultantes. Ambos grupos recibieron trombolíticos con tiempos puerta aguja similares: ACV $50 \pm 10,8 \mathrm{~min}$, CIMIC $52 \pm 15$ $\min (\mathrm{p}=0,2)$.

Las características de los pacientes con CIMIC que recibieron TIV, así como su pronóstico según mRS, se muestran en la Tabla 1.

El síntoma más frecuente de consulta en los enfermos con CIMIC fue la afasia global pura. El pronóstico a los 3 meses de los CIMIC que fueron trombolizados fue bueno, todos los pacientes estaban independientes a los 3 meses, con 8 de ellos absolutamente asintomáticos (mRS de $0)$. No se presentaron hemorragias sistémicas o intracraneanas en los pacientes con CIMIC que fueron tratados con TIV.

\section{Discusión}

Nuestro estudio demuestra que los CIMIC son frecuentes en una unidad de emergencia, de hecho, 1 de cada 6 pacientes que se presentaron en nues- tro Servicio de Urgencia con la sospecha de ACVi, resultó finalmente con diagnóstico de CIMIC. Estas cifras concuerdan con estudios previos en los que su porcentaje puede fluctuar entre 5 y $30 \%$ de los pacientes interpretados inicialmente como portadores de $\mathrm{ACVi}^{6-9}$. Al igual que en experiencias anteriores, la forma de presentación clínica más frecuente fue la de un cuadro afásico puro ${ }^{8}$.

En nuestra experiencia, por cada paciente con CIMIC tratado con TIV, 19 pacientes portadores de isquemias cerebrales recibieron tratamiento trombolítico, lo que corresponde solamente a $5 \%$ del total de pacientes tratados con TIV con diagnóstico final de CIMIC, cifra plenamente concordante con experiencias de otros grupos que describen cifras cercanas a $4,3 \%{ }^{8}$. No se presentaron complicaciones hemorrágicas sistémicas o cerebrales por el uso de TIV en nuestros casos con CIMIC, hecho que es concordante con un reciente estudio de Tsivgoulis et $\mathrm{al}^{17}$.

Considerando la baja frecuencia con la que pacientes con CIMIC reciben terapia de TIV, junto con su riesgo hemorrágico bajo y, por otra parte, tomando en cuenta de que claramente los pacientes ACVi se benefician de este tratamiento que es tiempo dependiente ${ }^{3}$, nuestra opinión es 
que debe privilegiarse en los servicios de urgencia la reducción del tiempo puerta aguja, por sobre tener un diagnóstico $100 \%$ definitivo y con imágenes de la presencia de un ACVi, debido a que esta acuciosidad diagnóstica con neuroimágenes puede además no lograrse en todos los pacientes ${ }^{12}$.

Nuestro estudio posee fortalezas al tratarse de un registro prospectivo de pacientes consecutivos en un ambiente clínico habitual y con un número significativo de enfermos consultantes y también con alta frecuencia de administración de TIV. Su limitación es que se trata de la experiencia de un solo centro, el cual es de alta complejidad, con turnos de urgencia cubiertos en su mayoría por neurólogos vasculares, lo que no necesariamente representa la realidad de otras instituciones del país.

En conclusión, la TIV en pacientes con cuadros imitadores de isquemia cerebral es poco frecuente y segura en términos de pronóstico funcional y riesgo de hemorragias.

\section{Referencias}

1. The National Institute of Neurological Disorders and Stroke rt-PA Stroke Study Group. Tissue plasminogen activator for acute ischemic stroke. N Engl J Med 1995; 333: 1581-7.

2. Hacke W, Kaste M, Bluhmki E, Brozman M, Dávalos A, Guidetti D, et al. Thrombolysis with alteplase 3 to 4.5 hours after acute ischemic stroke. N Engl J Med 2008; 359: 1317-29.

3. Emberson J, Lees KR, Lyden P, Blackwell L, Albers G, Bluhmki E, et al. Stroke Thrombolysis Trialists' Collaborative Group. Effect of treatment delay, age, and stroke severity on the effects of intravenous thrombolysis with alteplase for acute ischaemic stroke: a meta-analysis of individual patient data from randomized trials. Lancet 2014; 29: 1929-35.

4. Saver JL. Time is brain-quantified. Stroke 2006; 37: 2636.

5. Jauch EC, Saver JL, Adams HP Jr, Bruno A, Connors JJ, Demaerschalk BM, et al. American Heart Association Stroke Council; Council on Cardiovascular Nursing; Council on Peripheral Vascular Disease; Council on Clinical Cardiology. Guidelines for the early manage- ment of patients with acute ischemic stroke: a guideline for healthcare profession-als from the American Heart Association/American Stroke Association. Stroke 2013; 44: 870-947.

6. Hand PJ, Kwan J, Lindley RI, Dennis MS, Wardlaw JM. Distinguishing between stroke and mimic at the bedside: the Brain Attack Study. Stroke 2006; 37: 769-75.

7. Brunser AM, Illanes S, Lavados PM, Muñoz P, Cárcamo D, Hoppe A, et al. Exclusion criteria for intravenous thrombolysis in stroke mimics: an observational study. J Stroke Cerebrovasc Dis 2013; 22: 1140-5.

8. Winkler DT, Fluri F, Fuhr P, Wetzel SG, Lyrer PA, Ruegg S, et al. Thrombolysis in stroke mimics: frequency, clinical characteristics, and outcome. Stroke 2009; 40: 1522-5.

9. Libman RB, Wirkowski E, Alvir J, Rao T. Conditions that mimic stroke in the emergency department. Arch Neurol 1995; 52: 1119-22.

10. Mouradian MS, Rodgers J, Kashmere J, Jickling G, McCombe J, Emery DJ, et al. Can rt-PA be administered to the wrong patient? Two patients with somatoform disorder. Can J Neurol Sci 2004; 31: 99-101.

11. Brown DL, Barsan WG, Lisabeth LD, Gallery ME, Morgenstern LB. Survey of emergency physicians about recombinant tissue plasminogen activator for acute ischemic stroke. Ann Emerg Med 2005; 46: 56-60.

12. Brunser AM, Hoppe A, Illanes S, Díaz V, Muñoz P, Cárcamo D, et al. Accuracy of diffusion-weighted imaging in the diagnosis of stroke in patients with suspected cerebral infarct. Stroke 2013: 44; 1169-71.

13. GUSTO Investigators. An international randomized trial comparing four thrombolytic strategies for acute myocardial infarction. N Engl J Med1993; 329: 673-82.

14. Rankin J. Cerebral vascular accidents in patients over the age of 60. II. Prognosis. Scott Med J 1957; 2 (5): 200-15.

15. Farrell B, Godwin J, Richards S, Warlow C. The United Kingdom transient ischaemic attack (UK-TIA) aspirin trial: final results. J Neurol Neurosurg Psychiatry 1991; 54 (12): 1044-54.

16. Van Swieten JC, Koudstaal PJ, Visser MC, Schouten HJ, van Gijn J. Interobserver agreement for the assessment of handicap in stroke patients. Stroke 1988; 19 (5): 6047.

17. Tsivgoulis G, Zand R, Katsanos AH, Goyal N, Uchino $\mathrm{K}$, Chang J, et al. Safety of intravenous thrombolysis in stroke mimics: prospective 5-year study and comprehensive meta-analysis. Stroke 2015; 46: 1281-7. 